\title{
A Comparative Study on Online Teaching Practices in China and South Korea in the Time of COVID-19
}

\author{
Shi Yu ${ }^{1}$, Bing Xie ${ }^{2}$ \\ ${ }^{1}$ China University of Mining and Technology, School of Chemical Engineering, \\ No. 1 Daxue Road, Xuzhou, Jiangsu Province, P. R. China \\ shiyu_cumt@163.com \\ ${ }^{2}$ School of Finance, Xuzhou University of Technology, \\ No. 2 Lishui Road, Yunlong Dist., Xuzhou, Jiangsu Province, P. R. China \\ bingxie_xzit@163.com
}

\begin{abstract}
The transition from traditional teaching practices to remote education was sped up by the outbreak of COVID-19 pandemic dramatically. The international students who have to stay in their home countries due the travel restrictions rely on the online study. In this manuscript, we examine how the teaching methodology affects the outcome of remote education (e.g., an undergraduate program provided by CUMT, and a graduate program provided by Gachon University). Both SPOC and online streaming classes were evaluated based on the performance of international undergrads majored in chemical engineering. And multiscale simulations were adopted to elucidate the complex transport phenomena in chemical engineering classes to develop a more efficient method for distant engineering education. The preliminary results of this comparative study on online teaching practices indicate that active studies play an important role in online study for international students.
\end{abstract}

Keywords: Online teaching, Planning for teaching, International students, COVID-19 pandemic.

\section{Introduction}

The COVID-19 pandemic has significant impacts on the education sector globally, since the classroom routines and schedules are disrupted. There were nationwide school and university closures in more than 100 countries in 2020 [1]. As a result, although distant education has a long history [2], the transition from traditional course to online course is sped up by school closures abruptly. Specifically, online study plays an essential role for international students who have to stay in their home countries due to travel restrictions.

As the COVID-19 pandemic is promoting remote teaching for both domestic and international students, more and more studies on online teaching practices have been performed to improve the current remote teaching model. Recently, C. M. Domhnaill et al. [1] studied how the remote education is affected by home broadband and student engagement systematically. Y. Ren reported the difficulties of online education for international students in University of International Business and Economics [3] that more than $40 \%$ undergraduate students were not very satisfied with online study in that specific university. Y. Zhang et al. [4] performed an analysis on how the daily management of international students are affected by COVID-19 pandemic.

In addition, the COVID-19 pandemic has quite different effects on different courses. An extreme case is to teach wet bench laboratory contents through online education [5]. Obviously, it is extremely challenging to conduct experiments remotely, cause such laboratory courses rely on hands-on experiences which are hard to deliver through internet. Therefore, multiscale simulations are adopted as an alternative for such laboratory courses [5]. Nowadays, as simulations at various scales become a standard toolbox for academic research, it is also widely used in undergraduate education. More traditional courses have been transitioned to remote data analyses, virtual simulations, literature reviews, and online discussions [6]. Hence, it is of great interest to investigate how the remote teaching of technical courses can be affected by different teaching methods, especially the multiscale simulations, since it is easier for students to carry out simulations online than performing experiments remotely. The number of international students enrolled in universities in China kept on increasing before the breakout of the COVID-19 pandemic. The majority of them were largely affected by the pandemic and shifted to online study. In this manuscript, we focus on the online teaching of chemical engineering course in China University of Mining \& Technology (CUMT). For comparison, we also perform a case study on a graduate program provided by Gachon University in South Korea. Due to time zone differences, both SPOC (small private online course) which can be accessed by international students at any time as well as online streaming classes are provided to the international undergraduate students majored in chemical engineering in CUMT, which allows us to investigate how the different forms of online study affect the outcome of distant education. On the other hand, since the predominant method of student instruction in chemical engineering classes remains the transmission model that the instructor's role is to transmit information as a quantifiable resource to students [7], we also seek to examine the effects of active learning such as idea pitches, project presentations which are frequently used in business schools on remote education.

\section{The Influence of COVID-19 Pandemic on Number of International Students in China}

During last two decades, the number of international students in universities in China grew rapidly before 2019, as 
demonstrated in Figure 1a which is based on datasets provided by Ministry of Education of China [8]. And most international students come from Asia and Africa. As shown in Figure 1b, both the number of students who pursued academic degrees and the number of students who were funded by government exhibit approximately 10 -fold increase during last two decades. However, this increase in the number of international students was stopped by the outbreak of COVID-19. Although the data for the whole nation is still inaccessible, no new international student is recruited since 2020 in CUMT, due to the strict travel restrictions to suppress the spread of coronavirus. For those ungraduated international
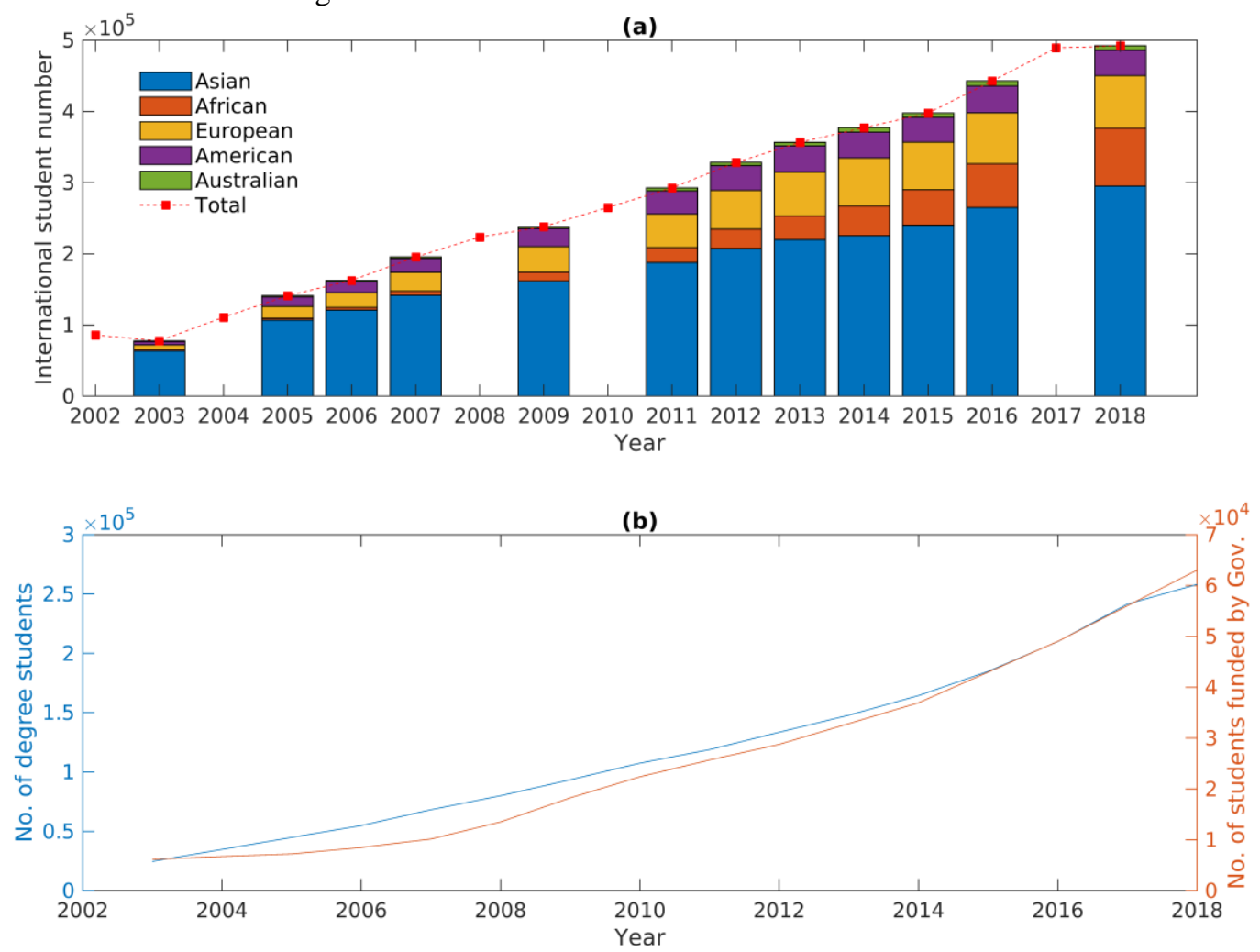

Figure 1: (a) The total number of international students for last two decades; (b) The number of students who purchased degrees or received government funding in China during last two decades.

\section{Effects of Teaching Methodology on Remote Education}

\subsection{Student Engagement}

Although many international students have to stay in their home countries for two years after the beginning of covid-19 pandemic, some international students remain on campus. To minimize the impacts of pandemic on daily academic activities, both online course for international students who are currently abroad and traditional course for students on campus are provided at the same time. Therefore, the students on campus can be considered as an ideal control group to evaluate the performance of students who take online course.

With the rapid development of Information Communication Technologies (ICTs), both MOOC [9] (massive online open courses) and SPOC (small private online course) were adopted by many universities even before the pandemic. For international students majored in Chemical Engineering in CUMT, both SPOC and online streaming classes are provided. As shown in Figure 2, the attendance rates for traditional students who are currently living in their home countries, a transition from traditional course to online course is required.

For a large fraction of international students who pursue degrees in Chinese universities, they prefer technical courses taught in English rather than in Chinese. Therefore, the teaching experiences in Chinese for domestic students cannot be reused in the courses developed for international students directly. Hence, it is crucial to figure out the effective and efficient method of remote education for international students during the pandemic. (a)

classroom teaching, SPOC, and online streaming classes are plotted for last three years. As can be seen from Figure 2, the attendance rate for SPOC is the lowest, which is approximately one half of that for traditional course and online streaming course. And the attendance rate for classroom teaching is similar as that for online streaming classes. These observations demonstrate that international students are still willing to learn the technical course by traditional method. For the SPOC, the undergraduate students are not able to communicate with instructors in real time. Therefore, they consider the SPOC to be less effective that they are reluctant to take the SPOC course. Although for SPOC, undergraduate students have more freedom that they can decide when to watch the video clips uploaded online and they can revisit those study materials multiple times after the class. Nevertheless, it turns out that those undergraduate students rarely revisit the SPOC sites to review the covered contents. Given this fact, to facilitate the online study of international students, especially those abroad, online discussion session seems to be necessary when SPOC is adopted. Otherwise, international students in SPOC might be less engaged compared to those students who sit in classroom. 


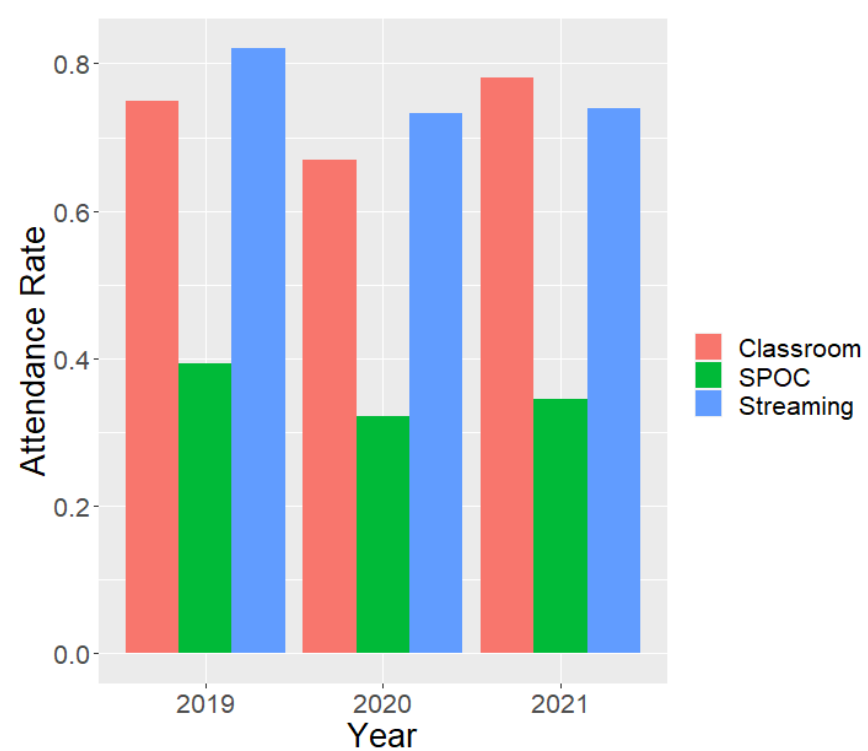

Figure 2: Attendance rates as a function of teaching method for last three years

\subsection{Simulation for Remote Education}

We chose the principles of chemical engineering taught in CUMT for international students as the example course to investigate the effects of applications of multiscale simulation techniques on distance education. Although the traditional principles of chemical engineering course focus on the macroscale transport phenomenon such as momentum transfer, mass transfer, and heat transfer, the microscale mechanism of those transport phenomenon can be elucidated with simulations at molecular scale [10-12].

As shown in Figure 3a, a 2D molecular dynamics simulation of surfactants with solvent particles was carried out to describe the diffusion at microscale, which allow the international students to have a better understanding of mass transfer. A similar simulation of collisions of hard spheres in gas phase can provide direct impression on kinetic theory, which can help the undergraduate students to better understand the heat transfer phenomena. Moreover, as depicted in Figure 3b, the famous Lees-Edwards boundary condition can be applied to the hard spheres systems with periodic boundary conditions to model the laminar flow. As the elementary fluid mechanics is critical for the principles of chemical engineering course, the visualization of Lees-Edwards boundary condition can be a good supplementary study material for this course. In addition, as CFD became a standard computational tool for industry as well as scientific research, a brief introduction of CFD computation within the principles of chemical engineering course allow the undergraduates to familiarize themselves with research tools which can be helpful for their graduate study. As demonstrated in Figure 3c, an example of velocity distribution of a 2D flow field is plotted. And the international students can revisit the important definition like vortex and vector field through this kind of CFD simulation.

Another advantage of application of simulations in online teaching is that international students pay more attention when video clips obtained by different simulations are included in classes, since the traditional classes with only equations and tables are not that attractive to international undergrads.

(a)

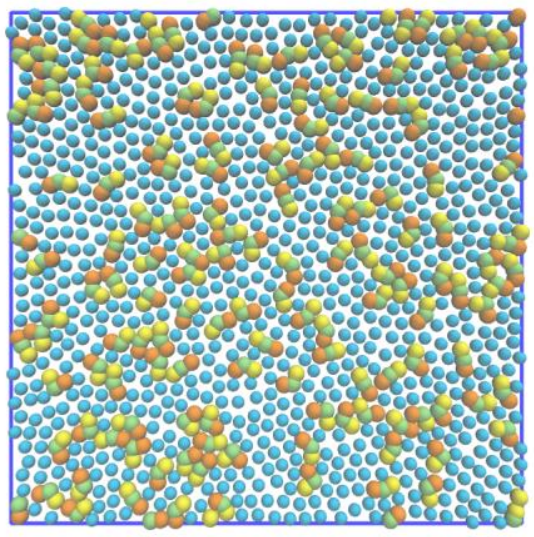

(b)

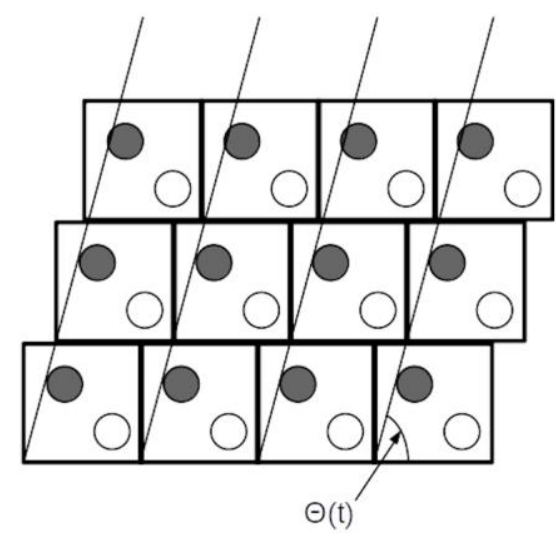

(c)

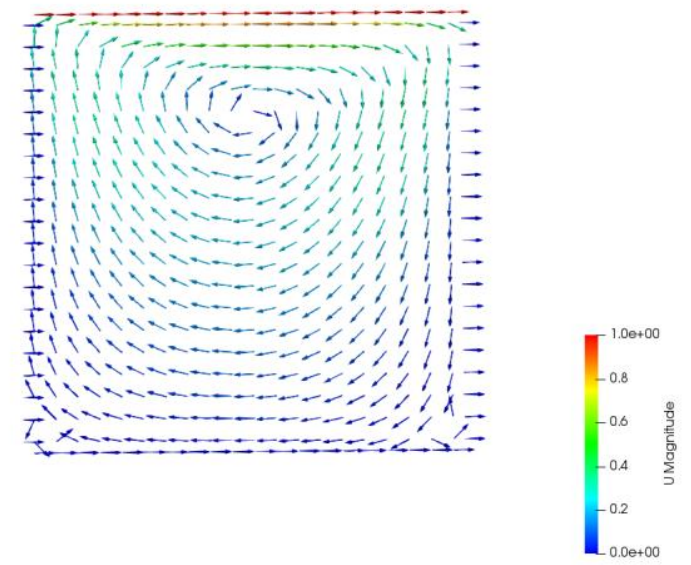

Figure 3: (a) A snapshot of a 2D molecular dynamics simulation of micelles with solvent particles; (b)

Lees-Edwards boundary condition; (c) Velocity distribution obtained by CFD calculations.

\subsection{Remote Education for a Korean Graduate Program}

For comparison, a graduate program provided by Gachon University was chosen as another example of remote education. For this specific DBA program provided by South Korean university to Chinese students, most students choose to take the online courses because of the severe pandemic. As can be seen from the Figure 4a, more graduate students are enrolled in this program since the outbreak of COVID-19, which is different from the trend that fewer international students are enrolled in universities in China. This difference might be attributed the facts that China and Korea are very close and this specific graduate program recruit students abroad without requiring them to be in South Korea. 
Therefore, to maintain the number of international students, remote education is necessary.

(a)

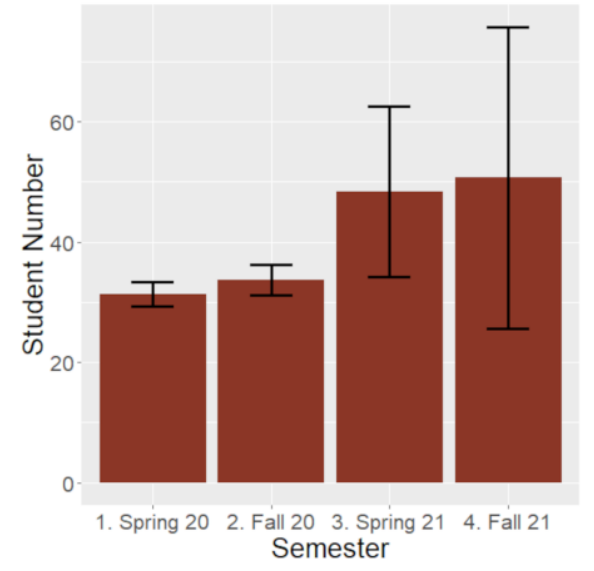

(b)

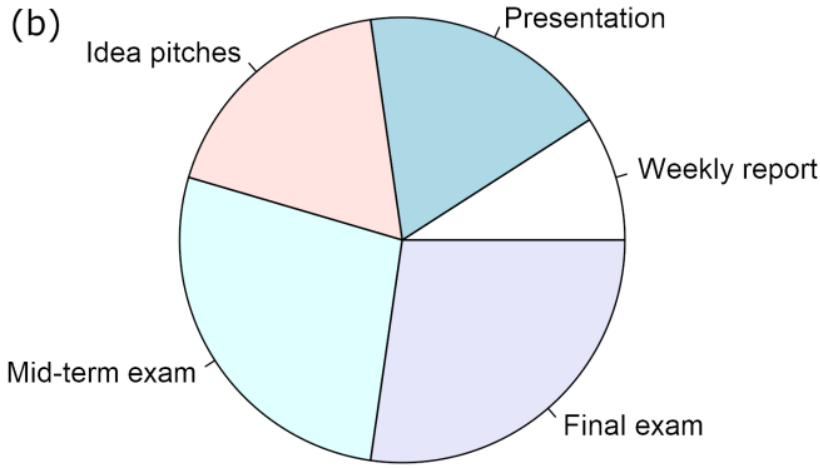

Figure 4: (a) Average number of students per class (The error bars are standard deviations); (b) Sample grading criteria.

Unlike technical courses for engineering students, this specific business program requires students to perform more active studies. As shown in Figure 4b, approximately $40 \%$ of the final grade is based on idea pitches, weekly report, and presentations for a business course. Students are divided into different groups to perform literature survey, online discussions, and final project presentations. One big advantage of this graduate program is that it is provided to Chinese students who can easily communicate and collaborate with each other. For the international program in Chinese universities, it is quite challenging for international students from different countries or even from different continents to collaborate with each other on some research projects.

The students enrolled in this graduate program are much more engaged compared to the undergraduate international students majored in chemical engineering in CUMT, as the attendance rates for this graduate program is always above $90 \%$. On one hand, the online discussion sessions are easier to carry out for business classes. On the other hand, complicated models, equations, and mathematical derivations which are necessary for technical engineering classes are not so interesting especially for online teaching practices. Besides, for this specific graduate program in Gachon University, all enrolled students are from China who have similar backgrounds, which is quite convenient for the instructors, as all students are used to the same pace. For undergraduate or graduate programs provided by Chinese universities, students are usually so diverse that it is really difficult to figure out an appropriate teaching strategy for every student.

\section{Conclusions}

Due to the outbreak of COVID-19, remote education becomes more and more important for teaching practices in universities, especially for international students. In this manuscript, we compared different forms of remote education, such as SPOC and online streaming. According to our limited datasets, international students prefer online streaming classes or traditional classes over SPOC. This finding confirms the importance of direct communications between instructor and students. Moreover, by comparing a CUMT international undergraduate program and a graduate program provided by Gachon University, we observed that active studies like online discussion, small group research projects play an important role in remote education that students are more engaged in such active learning. Besides, we have demonstrated several examples of applications of simulation techniques in chemical engineering classes teaching. Note that those simulations not only help to elucidate the complicated transport phenomena but also attract more international students during distant education. Through this comparative study on online teaching practices in China and South Korea in the time of COVID-19, we found that active study as well as more interesting teaching methodology (e.g., with multiscale simulations) are critical to remote education. In addition, instructors might have to pay more attention when SPOCs or MOOCs are adopted for online teaching, since students are less engaged when instructors are absent or when they cannot communicate with the instructors in real time.

\section{Acknowledgements}

We acknowledge support from China University of Mining \& Technology under CUMT teaching project grant 2021YB28.

\section{References}

[1] C. M. Domhnaill, G. Mohan, S. McCoy, "Home broadband and student engagement during COVID-19 emergency remote teaching," Distance Education, Vol. 42, No. 4, 465-493, 2021.

[2] B. Todhunter, "LOL-limitations of online learning-are we selling the open and distance education message short?" Distance Education, Vol. 34, No. 2, 232-252, 2013.

[3] Y. Ren, "Reflection on post pandemic online study for international students in China," Shanxi Youth, 18, 111-112, 2021

[4] Y. Zhang, L. Li, "The daily management of international students in the environment of normalized epidemic prevention and control," Journal of Hubei Open Vocational College, Vol. 33, No. 22, 61-62, 2020.

[5] M. Costabile, "Using online simulations to teach biochemistry laboratory content during COVID-19," Biochem Mol Biol Educ., Vol. 48, 5, 509-510, 2020.

[6] M. Arenas, "General considerations for online teaching practices in bioinformatics in the time of COVID-19," Biochem Mol Biol Educ., Vol. 49, 5, 683-684, 2021. 
[7] C. A. Bodnar, D. Anastasio, J. A. Enszer, D. D. Burkey, "Engineers at play: games as teaching tools for undergraduate engineering students," Journal of Engineering Education, Vol. 105, No. 1, 147-200, 2016.

[8] Ministry of Education, P. R. China. [Online]. Available: http://www.moe.gov.cn/jyb_xxgk/. [Accessed: Oct. 1, 2021].

[9] R. Corrado, E. Pretorius, G. Van Der Westhuizen, "Undergraduate students' experiences of the use of MOOCs for learning at a Cambodian University", Education Sciences, 11, 336, 2021.

[10] P. A. Craig, "Developing and applying computational resources for biochemistry education," Biochem Mol Biol Educ., Vol. 48, 6, 579-584, 2020.

[11] E. Karadoğan, F. Karadoğan, "Simulation-based learning modules for undergraduate engineering dynamics," Comput Appl Eng Educ., 27, 846-862, 2019.

[12] B. Spitznagel, P. R. Pritchett, T. C. Messina, M. Goadrich, J. Rodriguez, "An Undergraduate Laboratory Activity on Molecular Dynamics Simulations," Biochem Mol Biol Educ., Vol. 44, 2, 130-139, 2016.

\section{Author Profile}

Shi Yu works for China University of Mining \& Technology since 2018.

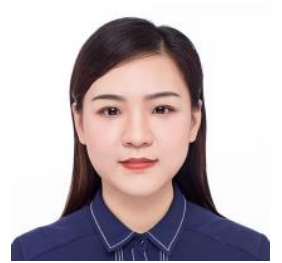

Bing Xie serves as the corresponding author of the article. She received the B.S. degree from China University of Mining \& Technology in 2012, and received the MSc degree in Marketing from INSEEC (Paris) in 2016. Since 2017, she works for Xuzhou University of Technology. And now she is pursuing a DBA degree in business in the Gachon University, South Korea. 